Egyptian Journal for Aquaculture

P-ISSN: 2090-7877

E-ISSN: 2636-3984

www.eja.journals.ekb.eg

Soha Ahmed, 2020; 10 (4):17-37

DOI: $10.21608 /$ eja.2021.48430.1039

\title{
Impact of different pollutants on some water quality aspects of Lake Mariout, Egypt Soha Mahmoud Ahmed
}

1. Limnology Department Central Lab. For Aquaculture Research, Agriculture Research Center, Egypt.

Received: November 01, 2020; Accepted: December 22, 2020 published: 2020 Vol.10 (4):17-37

\section{ABSTRACT}

The present study was carried out in Mariout Lake, which considered an important natural resource for fish production in Alexandria governorate. Lake Mariout is surrounded by different sources of pollution, such as sewage, industrial and agriculture drainages. Therefore, the present study was conducted in five locations to evaluate the effects resulting from pollution on the physical and chemical properties of water. Water samples were collected seasonally to investigate the effect of different geographical seasons (winter, spring, summer and autumn) of 2019. Obtained results revealed that $\mathrm{pH}$ values reached an increased value of 8.8. The highest values of total alkalinity and total hardness were 465.8 and $5400\left(\mathrm{mg} / \mathrm{l}\right.$ as $\left.\mathrm{CaCO}_{3}\right)$, respectively. The highest values of $\mathrm{NO}_{2}, \mathrm{NO}_{3}$ and total nitrogen that recorded alongside the study period were $0.2,2.32$ and $3.98 \mathrm{mg} / \mathrm{l}$, respectively. The highest recorded values of $\mathrm{PO}_{4}$ and total phosphorous were 0.42 and $0.92 \mathrm{mg} / \mathrm{l}$, respectively. Depending on the increased values of nutrients and chlorophyll 'a' as well as dissolved solids, some basins of the lake classified as eutrophic while the other basins classified as hypertrophic.

This study recommends urgently, starting on treating various types of wastewater before discharging it into the lake to reduce pollution rates and improve its water quality.

Keywords: Lake Mariout, pollution, physical and chemical properties of water, basins and geographical seasons

\section{Introduction}

Lake Mariout, for a long time, represents a source of fish production in Egypt. Currently, some parts of the lake are used in aquaculture activities. It is one of the most heavily populated urban areas in Egypt (Mateo 2009; 
Saad et al. 2017). The lake acts as a receptor for agricultural drainage and domestic wastewater. By the end of the $19^{\text {th }}$ Century, the development of irrigation systems of the adjacent fields made of Mariout Lake an intermediate water body to receive the excess of water from the irrigation channels, then the water was pumped out to the Alexandria Bay (Mateo, 2009).

The main drains and canal are Qalaa drain, El-Ummum drain, and Nubaria canal. The water inflow to the lake comes mainly from these sources plus those from the East and West treatment plants began in 1993 to treat Alexandrian wastewater. Both are primarily treatment plants and they discharge their final effluents into the lake and from the petrochemical area, domestic sewage from the wastewater Treatment Plant at the north and El-Qalaa drain are discharging in the main basin of the lake. Current water characteristics of the lake are not suitable for aquaculture activities where the basin receives daily about 200,000$300,000 \mathrm{~m}^{3} /$ day of sewage and $400000 \mathrm{~m}^{3}$ / day of agricultural wastewater mixed with sewage. In addition, discharging about 25,000$35,000 \mathrm{~m}^{3} /$ day of raw sewage and some industrial wastes into the lake (Scapini, 2009). Gehan et al. (2018) reported that throughout the last four decades, the lake has been subjected to man-made changes in its morphometric features as its area has been reduced. Lake Mariout is highly polluted. It receives the drastic human impacts among the Egyptian lakes; consequently, it has been greatly deteriorated from a productive lake to a heavily polluted basin, deeply suffering from eutrophication. Eutrophication has become a significant worldwide problem, which catastrophically affects aquaculture and local economies (Richlen $\boldsymbol{e t}$ al. 2010). The lake has been subjected to various anthropogenic activities, those activities greatly accelerated eutrophication process by increasing the rate of nutrient input in a water body, as rapid urbanization, industrialization, and intensifying of agricultural production.

Therefore, the aim of this work is to demonstrate the effects resulting from pollution on the physical and chemical properties of water in Lake Mariout, Egypt

\section{Material and methods}

\section{Study area}

Lake Mariout is located in the north of Egypt southeast to Alexandria City, belonging to the Nile River Delta and located between longitude $29^{\circ}$ $47.1^{\prime}$ to $29^{\circ} 50.4^{\prime} \mathrm{E}$ and latitude $31^{\circ} 7.5^{\prime}$ to $31^{\circ} 9^{\prime} \mathrm{N}$ (Saad $\boldsymbol{e t}$ al., 2017). 
The Lake is a shallow brackish water basin; its area is less than $65 \mathrm{~km}^{2}$, its water depth ranges from 1 to $3 \mathrm{~m}$.

\section{Sampling sites}

Figure (1) showing a schematic map of the lake, indicating different sampling sites which investigated during the present study, which representing the main basins of the lake as follow:

Fish Farm (Basin 1000 feddan): which receives its water from the drainage waters of El-Ummum (Site 1, drainage water) and El-Qallaa drains (Site 2, agricultural waste water).

Southwest Basin (Basin 2000 feddan): Site 1, Noubaria Canal which discharges wastewaters at the southern side of the basin and Site 2, Mogama industrial waste water into the Lake, which is discharged industrial effluents of eight factories were Salt and Soda, Extracted Oils, National Paper, Starch and Yeast, Nile Matches, South Alexandria Mills and Alexandria Foundry.

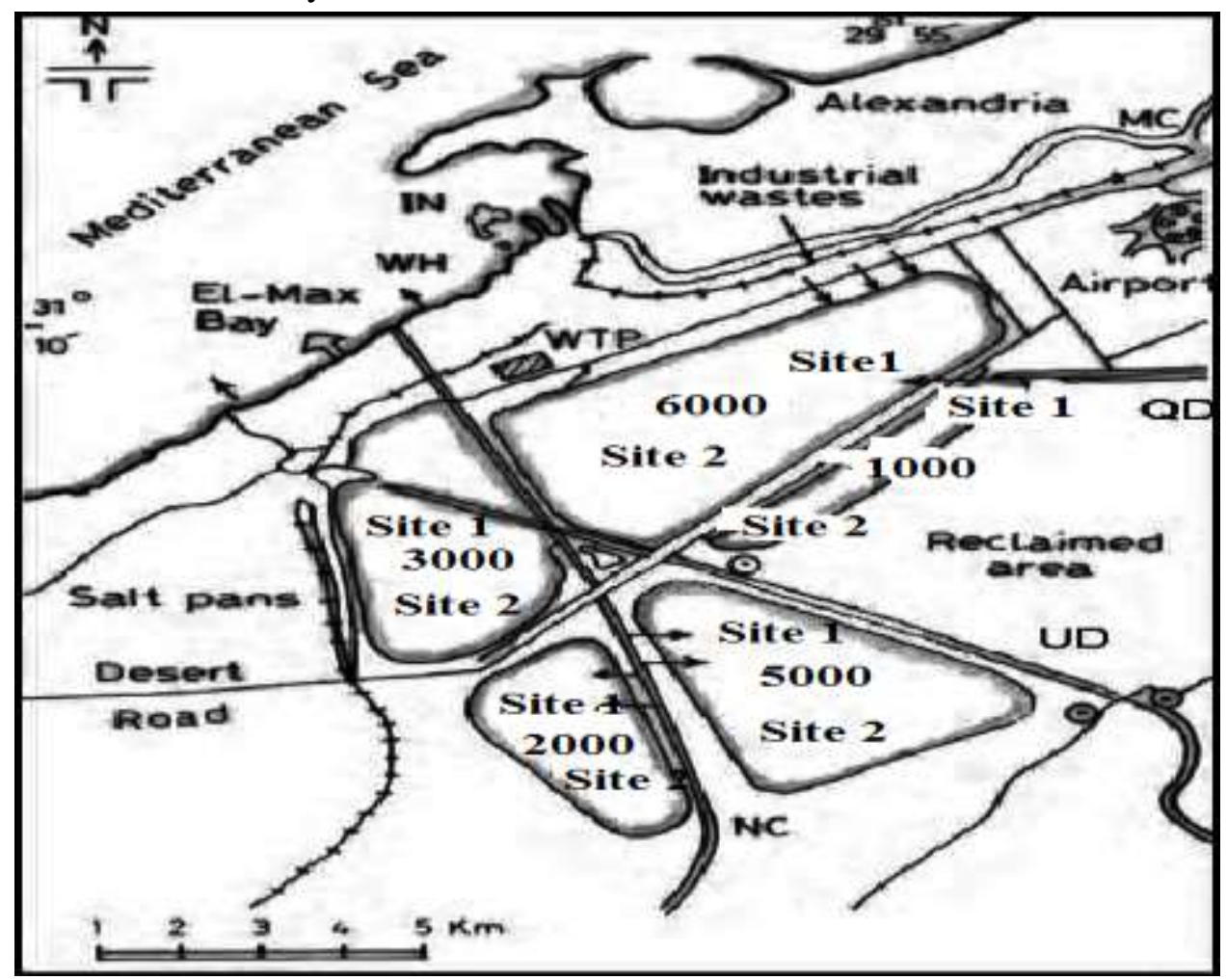

Fig. (1). Schematic map of Mariout Lake defining different investigated sites, which representing the lake basins. 
Northwest Basin (Basin 3000 feddan): Site 1, Industrial drainage (Misr petroleum company Max) and Site 2, El-Ommum drain (agricultural waste water).

Southeast Basin (Basin 5000 feddan): Site 1 and Site 2: El-Ommum drain (agricultural waste water).

Main Basin (Basin 6000 feddan): Site 1, El-Qallaa Drain Outfall, which discharges about $400000 \mathrm{~m}^{3}$ / day of agricultural waste water and sewage into the lake after primary treatment. In general, El-Qallaa Drain is considered the major source of pollution in the lake. The West Treatment Plant Outfall, which discharges about $200000-300000 \mathrm{~m}^{3}$ / day of settled sewage into the lake and Site 2, Noubaria Canal which discharges wastewaters at the western side of the basin.

\section{Analytical techniques:}

Water temperature $\left(\mathrm{T},{ }^{\circ} \mathrm{C}\right)$ was measured by oxygen meter (Hana HI 9142). Hydrogen ion concentration $(\mathrm{pH})$ was measured by $\mathrm{pH}$ meter (EUTECH instruments PCS tester 35). Total alkalinity (T. Alk., mg/l) and total hardness $(\mathrm{TH}, \mathrm{mg} / \mathrm{l})$ were determined by titration method. Electrical conductivity (EC), salinity and total dissolved solids (TDS) were measured by using a salinity-conductivity meter (model YSI Environmental, EC 300). Nitrite $\left(\mathrm{NO}_{2}, \mathrm{mg} / \mathrm{l}\right)$ was measured by diazodyzing method, while Nitrate $\left(\mathrm{NO}_{3} \mathrm{mg} / \mathrm{l}\right)$ and total nitrogen $(\mathrm{mg} / \mathrm{l})$ were measured by phenol di-sulphonic acid method. Total phosphorous $(\mathrm{mg} / \mathrm{l})$ and orthophosphates $(\mathrm{mg} / \mathrm{l})$ were measured by ascorbic acid method. Chlorophyll " $\mathrm{a}$ " concentration $(\mu \mathrm{g} / \mathrm{l})$ was assessed photometrically according to Vollenweider (1969). All photometric measurements were carried out by using spectrophotometer (model, WPA Linton Cambridge, UK) the all mentioned methods were according to APHA (2000).

\section{Statistical analysis:}

The obtained data were analyzed by using the GLM procedure with three-way analysis of variance (SAS, 2009), differences among means were tested for significance according to Duncan's multiple range test (Duncan, 1955). The following model was used to analyze the obtained data.

$Y_{i j k}=\mu+B_{i}+L_{j}+S_{t}+\left(B_{i} \times L_{j} \times S_{t}\right)_{i j t}+e_{i j t k}$

Where

$\mathrm{Y}_{\mathrm{ijk}}=$ Observation

$\mu=$ the overall mean, 
$\mathrm{B}_{\mathrm{i}}=$ Effect of Basin,

$\mathrm{L}_{\mathrm{j}}=$ Effect of Site,

$\mathrm{S}_{\mathrm{t}}=$ Effect of Season,

$\left(B_{\mathrm{i}} X L_{\mathrm{j}} \mathrm{X} \mathrm{S}_{\mathrm{t}} \mathrm{ijt}=\right.$ Interaction between Basin, Site and Season

$\mathrm{e}_{\mathrm{ijtk}}=$ random error.

\section{Results and Discussion}

\section{Water temperature:}

Water temperature has a direct and an indirect impact on fish productivity; warm water is much less capable of holding oxygen gas than colder water, which consequently may hamper fish production (FrancisFloyd 2003). The growth of tilapia in Lake Mariout may be acceptable between 20 and $35^{\circ} \mathrm{C}$ (El-Sherif and El-Feky 2009).

Water temperature values at the examined five sites during the study period are given in Table (1). The water temperature $\left({ }^{\circ} \mathrm{C}\right)$ attained its maximum value of $30.5^{\circ} \mathrm{C}$ during summer at (Northwest Basin and Main Basin) at site 2 and its minimum value of $15.6^{\circ} \mathrm{C}$ during winter at (Fish Farm) at site 2 . It could be observed that there were no significant differences among different sampling sites during the same season, referring that the differences refers to normal seasonal variations.

The annual average of water temperature, as shown in Fig. (2) was between $22.9{ }^{\circ} \mathrm{C}$ in (basin 1000) and $24.25{ }^{\circ} \mathrm{C}$ in (Main Basin).

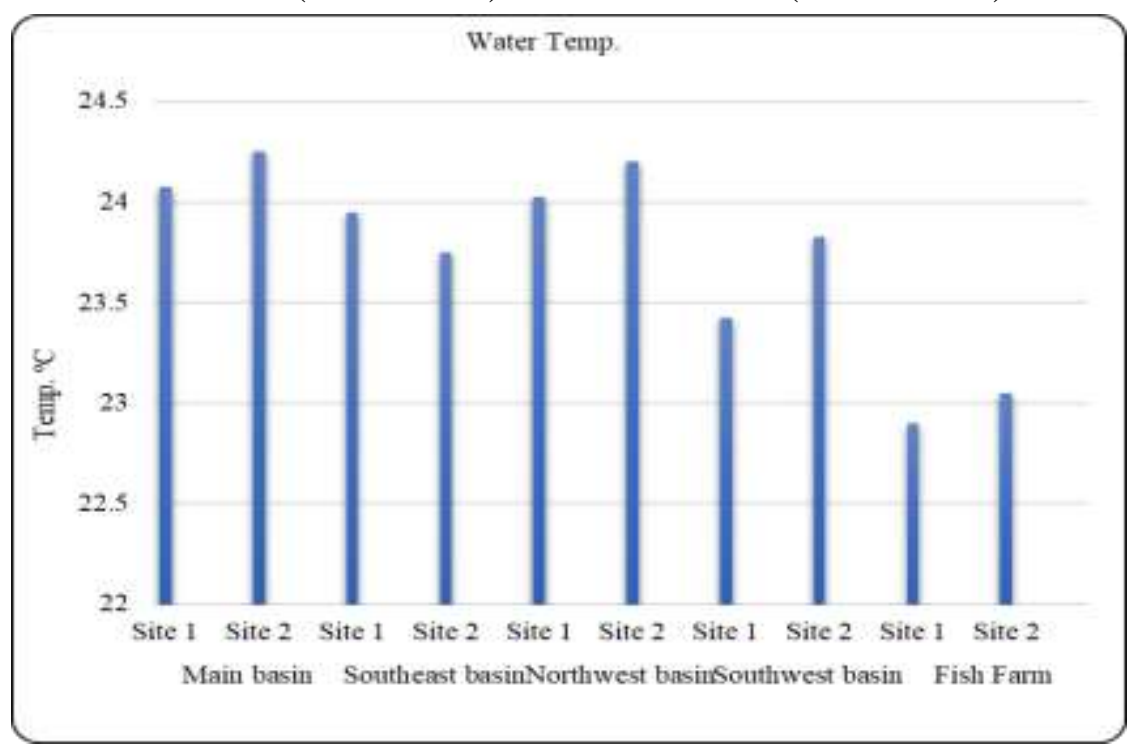

Fig. (2). The annual average of water temperature $\left({ }^{\circ} \mathrm{C}\right)$ at different sampling sites. 
Table (1). Some physico-chemical characteristics in different investigated sites during different seasons.

\begin{tabular}{|c|c|c|c|c|c|c|c|c|c|}
\hline Basins & & Seasons & $\begin{array}{c}\text { Temp } \\
\text { (C) }\end{array}$ & pH & $\begin{array}{c}\text { Salinity } \\
\%\end{array}$ & $\begin{array}{c}\text { EC } \\
\text { (mlmhos) }\end{array}$ & $\begin{array}{c}\text { TDS } \\
(\mathrm{mg} / \mathrm{l})\end{array}$ & $\begin{array}{c}\text { T. Alk } \\
(\mathrm{mg} / \mathrm{l} \text { as } \\
\left.\mathrm{CaCO}_{3}\right)\end{array}$ & $\begin{array}{c}\text { T. Har } \\
\text { (mg/l as } \\
\left.\mathrm{CaCO}_{3}\right)\end{array}$ \\
\hline \multirow{8}{*}{$\begin{array}{l}\Xi \\
\Xi \\
\Xi \\
\Xi \\
\Xi \\
\Sigma\end{array}$} & \multirow{4}{*}{ 氙 } & Winter & $16.5^{\mathrm{g}}$ & $8^{\mathrm{NS}}$ & $3^{\mathrm{d}}$ & $6.76^{\mathrm{ef}}$ & $3.86^{\mathrm{d}}$ & $245^{\mathrm{g}}$ & $1850^{\circ}$ \\
\hline & & Spring & $23.3^{\text {ef }}$ & 8.4 & $3.3^{\mathrm{d}}$ & $5.73^{\mathrm{f}}$ & $2.87^{\mathrm{e}}$ & $295^{\mathrm{f}}$ & $2050^{\mathrm{n}}$ \\
\hline & & Summer & $30.1^{\mathrm{a}}$ & 8.6 & $3.7^{\mathrm{c}}$ & $7.1^{\mathrm{d}}$ & $3.6^{\mathrm{d}}$ & $275^{\mathrm{fg}}$ & $3400^{g}$ \\
\hline & & Autumn & $26.4^{\mathrm{c}}$ & 7.91 & $3.4^{\mathrm{d}}$ & $5.6^{\mathrm{f}}$ & $3.3^{\mathrm{de}}$ & $350^{\text {de }}$ & $1250^{\mathrm{v}}$ \\
\hline & \multirow{4}{*}{$\stackrel{N}{\stackrel{N}{*}}$} & Winter & $16.9^{\mathrm{g}}$ & 7.46 & $4.2^{\mathrm{bc}}$ & $7.85^{\mathrm{d}}$ & $4.08^{\mathrm{cd}}$ & $305^{\mathrm{e}}$ & $1850^{\circ}$ \\
\hline & & Spring & $22.9^{f}$ & 7.64 & $2.4^{\mathrm{e}}$ & $4.58^{\mathrm{g}}$ & $2.29^{\mathrm{e}}$ & $265^{\mathrm{fg}}$ & $2000^{\mathrm{n}}$ \\
\hline & & Summer & $30.5^{\mathrm{a}}$ & 8.23 & $4.4^{\mathrm{b}}$ & $9.1^{\mathrm{b}}$ & $4.5^{\mathrm{c}}$ & $250^{\mathrm{fg}}$ & $2950^{\text {hi }}$ \\
\hline & & Autumn & $26.7^{\mathrm{c}}$ & 8 & $3.3^{\mathrm{d}}$ & $6.43^{\text {ef }}$ & $3.1^{\mathrm{de}}$ & $315^{\mathrm{e}}$ & $1610^{\mathrm{rs}}$ \\
\hline \multirow{8}{*}{ 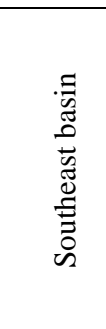 } & \multirow{4}{*}{$\stackrel{\overrightarrow{0}}{:=}$} & Winter & $16.7^{g}$ & 8.2 & $3^{\mathrm{d}}$ & $5.84^{\mathrm{f}}$ & $4.1^{\mathrm{cd}}$ & $272.2^{\mathrm{f}}$ & $1433^{t}$ \\
\hline & & Spring & $22.5^{\mathrm{f}}$ & 8.7 & $4.65^{\mathrm{b}}$ & $6.41^{\mathrm{ef}}$ & $4.95^{\mathrm{c}}$ & $328.8^{\mathrm{e}}$ & $1588^{\mathrm{s}}$ \\
\hline & & Summer & $30^{\mathrm{a}}$ & 8.8 & $4.9^{\mathrm{b}}$ & $7.9^{\mathrm{d}}$ & $4.94^{\mathrm{c}}$ & $361.7^{\mathrm{d}}$ & $2633^{j}$ \\
\hline & & Autumn & $26.6^{\mathrm{c}}$ & 8.5 & $4.74^{\mathrm{b}}$ & $6.88^{\mathrm{e}}$ & $4.85^{\mathrm{c}}$ & $390.1^{\mathrm{c}}$ & $1370^{\mathrm{u}}$ \\
\hline & \multirow{4}{*}{$\stackrel{N}{\stackrel{\Xi}{*}}$} & Winter & $16.5^{\mathrm{g}}$ & 8 & $4^{\mathrm{cd}}$ & $6.84^{\mathrm{e}}$ & $4.1^{\mathrm{cd}}$ & $369.9^{\mathrm{d}}$ & $1405^{\mathrm{t}}$ \\
\hline & & Spring & $22^{\mathrm{f}}$ & 8.2 & $4.24^{\mathrm{bc}}$ & $7.2^{\mathrm{de}}$ & $4.86^{\mathrm{c}}$ & $445.5^{\mathrm{ab}}$ & $1555^{\mathrm{s}}$ \\
\hline & & Summer & $30.2^{\mathrm{a}}$ & 8.8 & $4.65^{\mathrm{b}}$ & $7.92^{\mathrm{d}}$ & $4.75^{\mathrm{c}}$ & $415.3^{b}$ & $2580^{\mathrm{k}}$ \\
\hline & & Autumn & $26.3^{\mathrm{c}}$ & 8.3 & $4.05^{\mathrm{bc}}$ & $6.91^{\mathrm{e}}$ & $4.25^{\mathrm{cd}}$ & $465.8^{\mathrm{a}}$ & $1350^{\mathrm{u}}$ \\
\hline \multirow{8}{*}{ 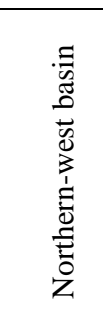 } & \multirow{4}{*}{ : } & Winter & $16.8^{\mathrm{g}}$ & 8.7 & $3.6^{\mathrm{c}}$ & $6.8^{\mathrm{e}}$ & $3.41^{\mathrm{de}}$ & $260^{f}$ & $1730^{\mathrm{q}}$ \\
\hline & & Spring & $23.5^{\text {ef }}$ & 8.05 & $3.5^{\mathrm{cd}}$ & $6.74^{\mathrm{e}}$ & $3.38^{\mathrm{de}}$ & $120^{j}$ & $3500^{\mathrm{f}}$ \\
\hline & & Summer & $30.1^{\mathrm{a}}$ & 8.2 & $3.7^{\mathrm{c}}$ & $7.48^{\mathrm{de}}$ & $3.8^{\mathrm{d}}$ & $275^{\mathrm{f}}$ & $2000^{\mathrm{n}}$ \\
\hline & & Autumn & $25.7^{d}$ & 8.5 & $4.8^{\mathrm{b}}$ & $7.73^{d}$ & $4.68^{\mathrm{c}}$ & $325^{\mathrm{e}}$ & $1420^{t}$ \\
\hline & \multirow{4}{*}{$\stackrel{N}{\stackrel{N}{*}}$} & Winter & $16.9^{g}$ & 7.5 & $4.99^{\mathrm{b}}$ & $9.5^{\mathrm{b}}$ & $4.77^{\mathrm{c}}$ & $185^{\mathrm{h}}$ & $2450^{1}$ \\
\hline & & Spring & $23.9^{\mathrm{e}}$ & 7.79 & $3.4^{\mathrm{cd}}$ & $6.47^{\mathrm{ef}}$ & $3.22^{\mathrm{de}}$ & $135^{\mathrm{ij}}$ & $3900^{\mathrm{e}}$ \\
\hline & & Summer & $30.5^{\mathrm{a}}$ & 8.1 & $3.6^{\mathrm{c}}$ & $7.1^{\mathrm{de}}$ & $3.6^{\mathrm{d}}$ & $260^{\mathrm{f}}$ & $3000^{\mathrm{h}}$ \\
\hline & & Autumn & $25.5^{\mathrm{d}}$ & 8.5 & $4.4^{\mathrm{bc}}$ & $7.43^{\mathrm{de}}$ & $4.63^{\mathrm{c}}$ & $335^{\mathrm{e}}$ & $1800^{\mathrm{p}}$ \\
\hline \multirow{8}{*}{ 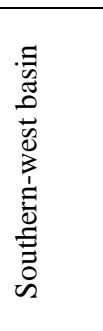 } & \multirow{4}{*}{ 氙 } & Winter & $16.5^{\mathrm{g}}$ & 8.03 & $4.1^{\mathrm{bc}}$ & $8.2^{\mathrm{cd}}$ & $4^{\mathrm{cd}}$ & $285^{\mathrm{f}}$ & $1820^{\circ p}$ \\
\hline & & Spring & $22^{f}$ & 8.04 & $4.3^{\mathrm{bc}}$ & $8.21^{\mathrm{cd}}$ & $4.11^{\mathrm{cd}}$ & $390^{\mathrm{c}}$ & $2235^{\mathrm{m}}$ \\
\hline & & Summer & $29.5^{\mathrm{b}}$ & 8.6 & $4.6^{\mathrm{b}}$ & $8.96^{\mathrm{c}}$ & $4.6^{\mathrm{c}}$ & $275^{\mathrm{f}}$ & $2783.33^{\mathrm{i}}$ \\
\hline & & Autumn & $25.7^{d}$ & 8 & $4.3^{\mathrm{bc}}$ & $7.52^{\mathrm{d}}$ & $4.75^{\mathrm{c}}$ & $355^{\mathrm{de}}$ & $1350^{\mathrm{u}}$ \\
\hline & \multirow{4}{*}{$\stackrel{N}{\stackrel{N}{0}}$} & Winter & $16.5^{\mathrm{g}}$ & 8.6 & $4.3^{\mathrm{bc}}$ & $8.5^{\mathrm{c}}$ & $4.2^{\mathrm{cd}}$ & $245^{g}$ & $2490^{1}$ \\
\hline & & Spring & $23.1^{\mathrm{ef}}$ & 8.7 & $4.2^{\mathrm{bc}}$ & $8.45^{\mathrm{c}}$ & $4.78^{\mathrm{c}}$ & $345^{\mathrm{de}}$ & $2660^{\mathrm{j}}$ \\
\hline & & Summer & $29.8^{b}$ & 8.8 & $4.6^{\mathrm{b}}$ & $8.5^{c}$ & $4.5^{\mathrm{c}}$ & $240^{g}$ & $3050^{\mathrm{h}}$ \\
\hline & & Autumn & $25.9^{d}$ & 8.5 & $4.8^{\mathrm{b}}$ & $7.73^{d}$ & $4.68^{c}$ & $325^{\mathrm{e}}$ & $1400^{\text {tu }}$ \\
\hline \multirow{8}{*}{ 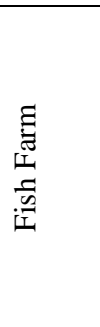 } & \multirow{4}{*}{ 离 } & Winter & $15.9^{\text {gh }}$ & 7.7 & $5.2^{\mathrm{a}}$ & $11^{\mathrm{a}}$ & $5.1^{\mathrm{bc}}$ & $275^{\mathrm{f}}$ & $1570^{\mathrm{s}}$ \\
\hline & & Spring & $22.4^{\mathrm{f}}$ & 7.94 & $5.6^{\mathrm{a}}$ & $10.62^{\mathrm{ab}}$ & $5.3^{\mathrm{bc}}$ & $345^{\mathrm{de}}$ & $5000^{\mathrm{b}}$ \\
\hline & & Summer & $29^{\mathrm{b}}$ & 8 & $5.8^{\mathrm{a}}$ & $11.5^{\mathrm{a}}$ & $5.6^{\mathrm{b}}$ & $150^{\mathrm{i}}$ & $4600.33^{c}$ \\
\hline & & Autumn & $24.3^{\mathrm{e}}$ & 8 & $5.7^{\mathrm{a}}$ & $11.2^{\mathrm{a}}$ & $5.6^{\mathrm{b}}$ & $355^{\mathrm{de}}$ & $1770^{\mathrm{q}}$ \\
\hline & \multirow{4}{*}{ 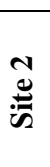 } & Winter & $15.6^{\mathrm{h}}$ & 7.5 & $5.3^{\mathrm{ab}}$ & $10.85^{\mathrm{ab}}$ & $5.37^{\mathrm{bc}}$ & $280^{\mathrm{f}}$ & $1490^{\text {st }}$ \\
\hline & & Spring & $22.8^{\mathrm{f}}$ & 8 & $5.4^{\mathrm{ab}}$ & $11.2^{\mathrm{a}}$ & $5.3^{\mathrm{bc}}$ & $265^{f}$ & $5400^{\mathrm{a}}$ \\
\hline & & Summer & $29.2^{b}$ & 8.3 & $6^{\mathrm{a}}$ & $11.43^{\mathrm{a}}$ & $6.23^{\mathrm{a}}$ & $350^{\mathrm{de}}$ & $4000^{\mathrm{d}}$ \\
\hline & & Autumn & $24.6^{\mathrm{e}}$ & 8 & $5.9^{\mathrm{a}}$ & $11.1^{\mathrm{a}}$ & $6^{\mathrm{a}}$ & $295^{\mathrm{f}}$ & $1680^{r}$ \\
\hline \multicolumn{3}{|c|}{$\pm \mathbf{S E}$} & 0.3 & 0.2 & 0.2 & 0.2 & 0.2 & 1.3 & 52.8 \\
\hline
\end{tabular}

Means in the same column having the same superscript letters are not significantly different $(\mathrm{P}<0.05)$. 
Table 1 continued

\begin{tabular}{|c|c|c|c|c|c|c|c|c|}
\hline Basins & & Seasons & $\begin{array}{c}\mathrm{NO}_{2} \\
(\mathrm{mg} / \mathrm{l})\end{array}$ & $\begin{array}{c}\mathrm{NO}_{3} \\
(\mathrm{mg} / \mathrm{l})\end{array}$ & $\begin{array}{c}\mathrm{TN} \\
(\mathrm{mg} / \mathrm{l})\end{array}$ & $\begin{array}{c}\mathrm{PO}_{4} \\
(\mathrm{mg} / \mathrm{l}) \\
\end{array}$ & $\begin{array}{c}\text { TP } \\
(\mathrm{mg} / \mathrm{l}) \\
\end{array}$ & $\begin{array}{c}\text { Chlo. "a" } \\
(\mu \mathrm{g} / \mathrm{l})\end{array}$ \\
\hline \multirow{8}{*}{ 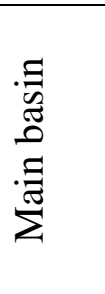 } & \multirow{4}{*}{ 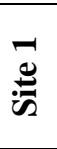 } & Winter & $0.02^{\mathrm{e}}$ & $0.09^{j}$ & $1.16^{\mathrm{h}}$ & $0.09^{\mathrm{e}}$ & $0.47^{\mathrm{cd}}$ & $268.6^{\mathrm{c}}$ \\
\hline & & Spring & $0.2^{\mathrm{a}}$ & $0.21^{\text {gh }}$ & $1.98^{\mathrm{ef}}$ & $0.4^{\mathrm{b}}$ & $0.53^{\mathrm{c}}$ & $193.7^{\mathrm{d}}$ \\
\hline & & Summer & $0.15^{\mathrm{ab}}$ & $1.35^{\mathrm{bc}}$ & $2.48^{\mathrm{d}}$ & $0.35^{\mathrm{b}}$ & $0.37^{\mathrm{de}}$ & $59.5^{\mathrm{n}}$ \\
\hline & & Autumn & $0.12^{\mathrm{ab}}$ & $0.31^{\mathrm{f}}$ & $1.61^{\mathrm{f}}$ & $0.4^{\mathrm{b}}$ & $0.47^{\mathrm{cd}}$ & $76.5^{1}$ \\
\hline & \multirow{4}{*}{ 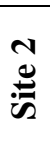 } & Winter & $0.09^{\mathrm{b}}$ & $0.63^{\mathrm{d}}$ & $1.31^{\mathrm{g}}$ & $0.4^{\mathrm{b}}$ & $0.92^{\mathrm{a}}$ & $68^{\mathrm{m}}$ \\
\hline & & Spring & $0.02^{\mathrm{e}}$ & $0.05^{\mathrm{k}}$ & $1.3^{\mathrm{g}}$ & $0.15^{\mathrm{cd}}$ & $0.35^{\mathrm{de}}$ & $115.6^{\text {gh }}$ \\
\hline & & Summer & $0.05^{\mathrm{cd}}$ & $0.31^{\mathrm{f}}$ & $2.79^{\mathrm{c}}$ & $0.21^{\mathrm{c}}$ & $0.21^{\mathrm{f}}$ & $71.4^{\mathrm{Im}}$ \\
\hline & & Autumn & $0.1^{\mathrm{b}}$ & $0.26^{\mathrm{g}}$ & $1.69^{f}$ & $0.19^{c}$ & $0.64^{b}$ & $47.6^{\circ}$ \\
\hline \multirow{8}{*}{ 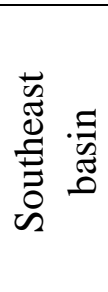 } & \multirow{4}{*}{ 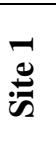 } & Winter & $0.02^{\mathrm{e}}$ & $1.24^{\mathrm{bc}}$ & $1.51^{\mathrm{f}}$ & $0.01^{\mathrm{h}}$ & $0.62^{b}$ & $79.07^{1}$ \\
\hline & & Spring & $0.04^{\mathrm{d}}$ & $0.36^{\mathrm{f}}$ & $0.88^{\mathrm{i}}$ & $0.06^{\mathrm{f}}$ & $0.69^{\mathrm{b}}$ & $110.5^{\mathrm{h}}$ \\
\hline & & Summer & $0.04^{\mathrm{d}}$ & $2.32^{\mathrm{a}}$ & $1.79^{\mathrm{ef}}$ & $0.05^{\mathrm{f}}$ & $0.49^{\text {cd }}$ & $100^{\mathrm{ij}}$ \\
\hline & & Autumn & $0.02^{\mathrm{e}}$ & $0.54^{\mathrm{e}}$ & $0.71^{j}$ & $0.06^{\mathrm{f}}$ & $0.53^{\mathrm{c}}$ & $89.8^{k}$ \\
\hline & \multirow{4}{*}{ 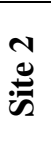 } & Winter & $0.03^{\mathrm{de}}$ & $0.98^{\mathrm{c}}$ & $1.63^{\mathrm{f}}$ & $0.03^{\mathrm{g}}$ & $0.56^{\mathrm{c}}$ & $87.7^{\mathrm{k}}$ \\
\hline & & Spring & $0.05^{\mathrm{cd}}$ & $0.06^{\mathrm{k}}$ & $0.96^{\mathrm{i}}$ & $0.06^{\mathrm{f}}$ & $0.78^{\mathrm{ab}}$ & $105.7^{\mathrm{i}}$ \\
\hline & & Summer & $0.06^{\mathrm{c}}$ & $1.35^{\mathrm{bc}}$ & $1.88^{\mathrm{ef}}$ & $0.07^{\mathrm{ef}}$ & $0.85^{\mathrm{a}}$ & $100.5^{\mathrm{ij}}$ \\
\hline & & Autumn & $0.03^{\mathrm{de}}$ & $0.78^{\mathrm{c}}$ & $0.93^{\mathrm{i}}$ & $0.07^{\mathrm{ef}}$ & $0.63^{\mathrm{b}}$ & $95.5^{\mathrm{j}}$ \\
\hline \multirow{8}{*}{ 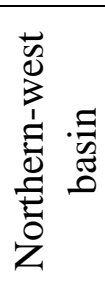 } & \multirow{4}{*}{ 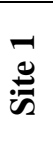 } & Winter & $0.09^{\mathrm{b}}$ & $0.85^{\mathrm{c}}$ & $1.05^{\mathrm{hi}}$ & $0.39^{\mathrm{b}}$ & $0.65^{\mathrm{b}}$ & $66.3^{\mathrm{m}}$ \\
\hline & & Spring & $0.05^{\mathrm{cd}}$ & $0.05^{\mathrm{k}}$ & $1.4^{\mathrm{g}}$ & $0.09^{\mathrm{e}}$ & $0.19^{\text {fg }}$ & $89.5^{\mathrm{k}}$ \\
\hline & & Summer & $0.03^{\mathrm{de}}$ & $0.27^{\mathrm{g}}$ & $3.9^{\mathrm{a}}$ & $0.42^{\mathrm{b}}$ & $0.65^{\mathrm{b}}$ & $59.4^{\mathrm{n}}$ \\
\hline & & Autumn & $0.08^{\mathrm{bc}}$ & $0.27^{\mathrm{g}}$ & $2.98^{\mathrm{bc}}$ & $0.07^{\text {ef }}$ & $0.51^{\mathrm{c}}$ & $87.5^{\mathrm{k}}$ \\
\hline & \multirow{4}{*}{$\begin{array}{c}\text { N } \\
\stackrel{0}{0}\end{array}$} & Winter & $0.02^{\mathrm{e}}$ & $0.35^{\mathrm{f}}$ & $1.5^{\mathrm{f}}$ & $0.08^{\mathrm{e}}$ & $0.41^{\mathrm{d}}$ & $414.8^{\mathrm{b}}$ \\
\hline & & Spring & $0.06^{\mathrm{c}}$ & $0.05^{\mathrm{k}}$ & $1.48^{\mathrm{f}}$ & $0.1^{\mathrm{d}}$ & $0.21^{\mathrm{f}}$ & $459.6^{\mathrm{a}}$ \\
\hline & & Summer & $0.03^{\mathrm{de}}$ & $1.78^{\mathrm{b}}$ & $3.14^{\mathrm{b}}$ & $0.1^{\mathrm{d}}$ & $0.14^{\mathrm{g}}$ & $130^{\mathrm{ef}}$ \\
\hline & & Autumn & $0.09^{\mathrm{b}}$ & $0.28^{\mathrm{g}}$ & $2.04^{\mathrm{e}}$ & $0.08^{\mathrm{e}}$ & $0.4^{\mathrm{d}}$ & $156.4^{\mathrm{e}}$ \\
\hline \multirow{8}{*}{ 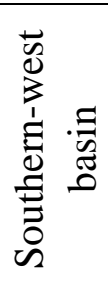 } & \multirow{4}{*}{$\underset{0}{\stackrel{3}{*}}$} & Winter & $0.05^{\mathrm{cd}}$ & $0.12^{\mathrm{i}}$ & $0.69^{j}$ & $0.2^{\mathrm{c}}$ & $0.28^{\mathrm{e}}$ & $80.6^{1}$ \\
\hline & & Spring & $0.04^{\mathrm{d}}$ & $0.17^{\mathrm{h}}$ & $0.48^{\mathrm{k}}$ & $0.08^{\mathrm{e}}$ & $0.3^{\mathrm{e}}$ & $135^{\mathrm{f}}$ \\
\hline & & Summer & $0.04^{\mathrm{d}}$ & $0.17^{\mathrm{h}}$ & $2.73^{\mathrm{c}}$ & $0.08^{\mathrm{e}}$ & $0.49^{\text {cd }}$ & $91.5^{j}$ \\
\hline & & Autumn & $0.08^{\mathrm{bc}}$ & $0.14^{\mathrm{i}}$ & $2.96^{\mathrm{bc}}$ & $0.08^{\mathrm{e}}$ & $0.37^{\mathrm{d}}$ & $62.9^{\mathrm{m}}$ \\
\hline & \multirow{4}{*}{ 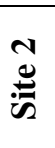 } & Winter & $0.09^{\mathrm{b}}$ & $0.12^{\mathrm{i}}$ & $1^{\text {hi }}$ & $0.07^{\mathrm{ef}}$ & $0.28^{\mathrm{e}}$ & $79.8^{1}$ \\
\hline & & Spring & $0.05^{\mathrm{cd}}$ & $0.13^{\mathrm{i}}$ & $0.87^{\mathrm{i}}$ & $0.05^{\mathrm{f}}$ & $0.17^{\mathrm{fg}}$ & $127.5^{\mathrm{g}}$ \\
\hline & & Summer & $0.19^{\mathrm{a}}$ & $0.19^{\mathrm{h}}$ & $1.05^{\mathrm{hi}}$ & $0.08^{\mathrm{e}}$ & $0.51^{\mathrm{c}}$ & $96.5^{j}$ \\
\hline & & Autumn & $0.08^{\text {bc }}$ & $0.27^{\mathrm{g}}$ & $3.98^{\mathrm{a}}$ & $0.07^{\mathrm{ef}}$ & $0.4^{\mathrm{d}}$ & $87.5^{\mathrm{k}}$ \\
\hline \multirow{8}{*}{ פ } & \multirow{4}{*}{ 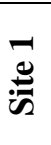 } & Winter & $0.05^{\mathrm{cd}}$ & $0.11^{\mathrm{i}}$ & $0.4^{\mathrm{k}}$ & $0.05^{\mathrm{f}}$ & $0.21^{\mathrm{f}}$ & $123.7^{\mathrm{g}}$ \\
\hline & & Spring & $0.02^{\mathrm{e}}$ & $0.15^{\text {hi }}$ & $1.19^{\mathrm{h}}$ & $0.06^{\mathrm{f}}$ & $0.29^{\mathrm{e}}$ & $260.8^{c}$ \\
\hline & & Summer & $0.02^{\mathrm{e}}$ & $0.17^{\mathrm{h}}$ & $1.7^{\mathrm{ef}}$ & $0.1^{\mathrm{d}}$ & $0.15^{\text {fg }}$ & $134.3^{\mathrm{f}}$ \\
\hline & & Autumn & $0.04^{\mathrm{d}}$ & $0.17^{\mathrm{h}}$ & $0.47^{\mathrm{k}}$ & $0.06^{\mathrm{f}}$ & $0.13^{\mathrm{g}}$ & $100.9^{\mathrm{ij}}$ \\
\hline & \multirow{4}{*}{ 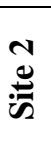 } & Winter & $0.05^{\mathrm{cd}}$ & $0.19^{\mathrm{h}}$ & $1.59^{\mathrm{f}}$ & $0.11^{\mathrm{d}}$ & $0.37^{\mathrm{d}}$ & $134.6^{\mathrm{f}}$ \\
\hline & & Spring & $0.02^{\mathrm{e}}$ & $0.26^{\mathrm{g}}$ & $2.4^{\mathrm{d}}$ & $0.1^{\mathrm{d}}$ & $0.42^{\mathrm{d}}$ & $275^{\mathrm{c}}$ \\
\hline & & Summer & $0.2^{\mathrm{a}}$ & $1.1^{\mathrm{a}}$ & $2.35^{\mathrm{d}}$ & $0.15^{\mathrm{cd}}$ & $0.59^{\mathrm{c}}$ & $130.9^{\mathrm{fg}}$ \\
\hline & & Autumn & $0.06^{\mathrm{c}}$ & $0.21^{\text {gh }}$ & $2.05^{\mathrm{e}}$ & $0.14^{\text {cd }}$ & $0.39^{d}$ & $119.5^{\text {gh }}$ \\
\hline \multicolumn{3}{|c|}{$\pm \mathbf{S E}$} & 0.002 & 0.007 & 0.01 & 0.001 & 0.002 & 1.04 \\
\hline
\end{tabular}

Means in the same column having the same superscript letters are not significantly different $(\mathrm{P}<0.05)$. 
However, this range of temperature in Lake Mariout water is suitable for Tilapia growth and is in agreement with the measured temperature obtained by Saad et al., 2017 (15.1 - 32.6) ${ }^{\circ} \mathrm{C}$.

\section{Hydrogen ion concentration $(\mathrm{pH})$ :}

Hydrogen ion concentration $(\mathrm{pH})$ plays an important role in many of the life processes in the sea; living organisms are very dependent on, and sensitive to $\mathrm{pH}$. It is dependent on the interaction of numerous substances dissolved in water, photosynthetic activity of aquatic plants, respiration of aquatic organisms, decomposition of organic matter, precipitation and/or dissolution of $\mathrm{CO}_{2}$ components and oxidation-reduction reactions (Tucker, 1984).

Obtained results of the study revealed that $\mathrm{pH}$ values alongside the study period ranged between 7.46 recorded in (Main Basin) at site 2 during winter, and 8.8 noticed in (Southwest Basin at site 2 and Southeast Basin at site 1 and 2) during summer. This is in agreement with the results obtained by El-Rayis et al. (2019), where Photosynthesis increases during the summer season. As shown in Fig. (3), the annual averages of $\mathrm{pH}$ values calculated at the end of the present study were between 7.83 in (Main Basin) and 8.65 in (Southern-west Basin).

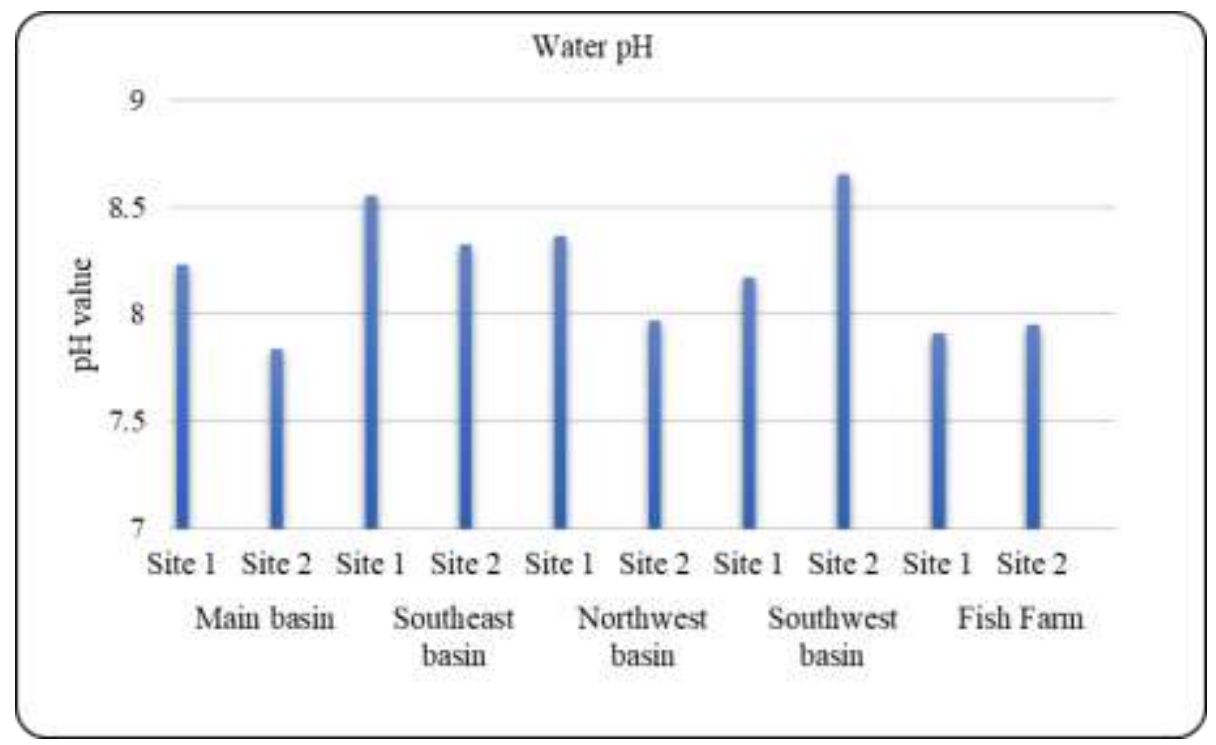

Fig. (3). The annual average of water $\mathrm{pH}$ at different sampling sites.

However all recorded values alongside the study period could be considered within the optimal range for fish culture, where safe level of $\mathrm{pH}$, for a normal fish productivity ranged between 5 and 9 and for 
maximum productivity, $\mathrm{pH}$ values ranged between 6.5 and 8.5 (Phang, 1991).

\section{Total dissolved solids (TDS):}

TDS includes those materials dissolved in the water, such as, bicarbonate, calcium, sulphate, magnesium, sodium, phosphate, nitrate, organic ions, and other ions. These ions are important in sustaining aquatic life. However, high concentrations can result in damage of organism's cell (Mitchell and Stapp, 1992). TDS values recorded alongside the present study period ranged between $2.29 \mathrm{mg} / \mathrm{l}$ in spring at (Main Basin at site 2) and $6.23 \mathrm{mg} / \mathrm{l}$ in summer at (Fish Farm at site 2). The annual average of TDS values was between 3.4075 and $5.725 \mathrm{mg} / \mathrm{l}$, in (Main Basin) and (Fish Farm), respectively (Fig. 4).

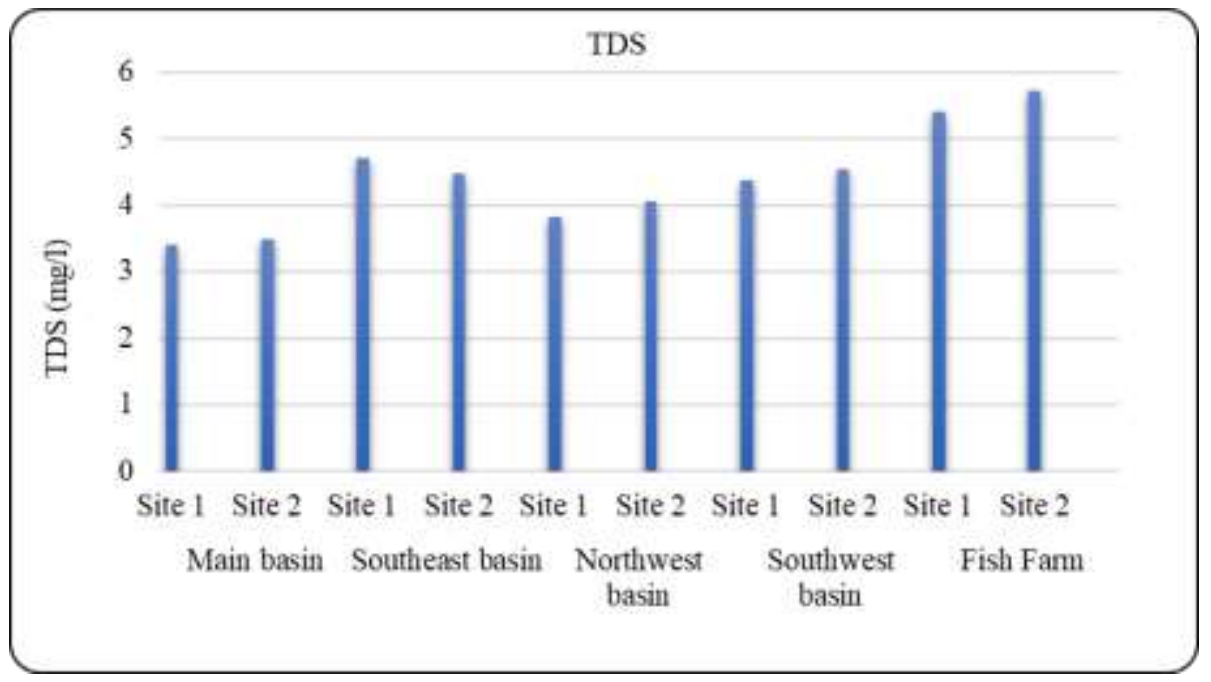

Fig. (4). The annual average of total dissolved solids (mg/l) at different sampling sites.

Similar values (2.56 - $5.6 \mathrm{mg} / \mathrm{l})$ previously obtained by Farouk $\boldsymbol{e t}$ al . (2020). However, these values are far greater than the standard value of $0.13 \mathrm{mg} / \mathrm{l}$ recommended by Davis $(\mathbf{1 9 9 3})$.

\section{Electric conductivity (EC)}

Electric conductivity reflects the quantities of dissolved salts and salinity (Thompson et al., 2012). EC values recorded during the present study ranged between 4.58 ( $\mathrm{ml}$ mhos) during spring at (Main Basin) and 11.5 (ml mhos) during summer at (Fish Farm). As illustrated in Fig. (5), the annual average of water electric conductivity calculated at the end of the present study was between 6.2975 and 11.145 ( $\mathrm{ml} \mathrm{mhos)} \mathrm{in} \mathrm{(Main}$ Basin) and (Fish Farm), respectively. 


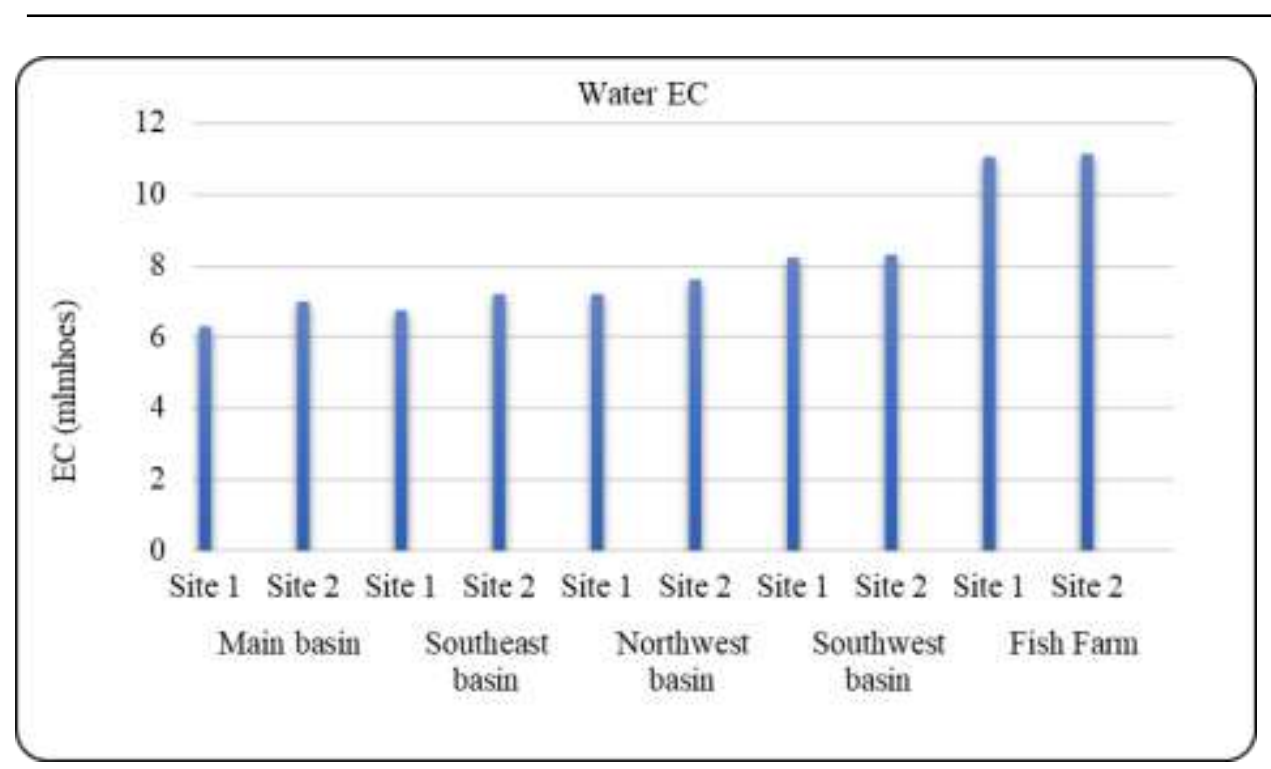

Fig. (5). The annual average of total EC ( $\mathrm{ml} \mathrm{mhos)} \mathrm{at} \mathrm{different} \mathrm{sampling} \mathrm{sites.}$

\section{Water Salinity}

It is observed that water salinity had the same trend of both TDS and EC. Its values ranged between $2.4 \%$, which recorded during spring at (Main Basin at site 2) and $6 \%$ during summer at (Fish Farm at site 2). As shown in Fig. (6), the annual average of water salinity ranged between 3.35 and $5.65 \%$, in (Main Basin) and (Fish Farm), respectively. Similar results mentioned by Farouk et al. (2020) who reported that the values of TDS, EC and salinity were higher in (basin 1000) than other basins.

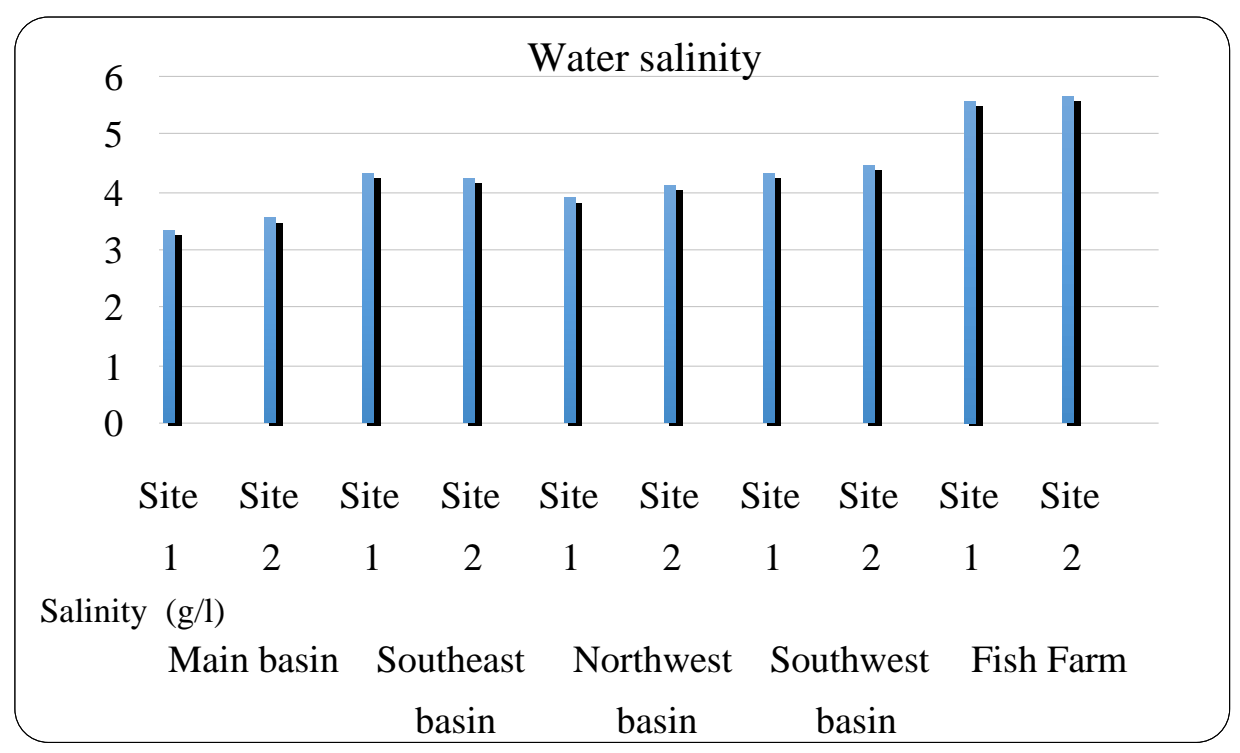

Fig. (6). The annual average of water salinity \%o at different sampling sites. 


\section{Total alkalinity (T.Alk):}

The Alkalinity may be defined as the excess of anions weak acids in seawater. Alkalinity is a measure of the capacity of the substances dissolved in the bicarbonate and carbonate which are ions formed when carbon dioxide or carbonate rocks dissolve in water. In other words, alkalinity is expressed as the sum of equivalents of $\mathrm{HCO}_{3}, \mathrm{CO}_{3}$ and $(\mathrm{OH})$ ions. The importance of alkalinity lies in its role in $\mathrm{CO}_{2}$ chemistry, trace metal speciation, buffer capacity of water, as a tracer for studying mixing processes between different water masses and a useful additional variable to study hydrographical features. It is affected by several processes, which are photosynthesis and respiration, nitrification, denitrification, sulfide oxidation, sulfide reduction, and $\mathrm{CaCO}_{3}$ dissolution. Recorded total alkalinity values at the end of the present work ranged between 120 $\mathrm{mg} / \mathrm{l}$ during spring at Northwest Basin at site 1 and $465.8 \mathrm{mg} / \mathrm{l}$ during autumn at Southeast Basin at site 2. As shown in Fig. (7), the annual average of total alkalinity values during the present study ranged between $228.75\left(\mathrm{mg} / \mathrm{l}\right.$ as $\left.\mathrm{CaCO}_{3}\right)$ in (Northern-west Basin) and $424.125(\mathrm{mg} / \mathrm{l}$ as $\mathrm{CaCO}_{3}$ ) in (Southeast Basin).

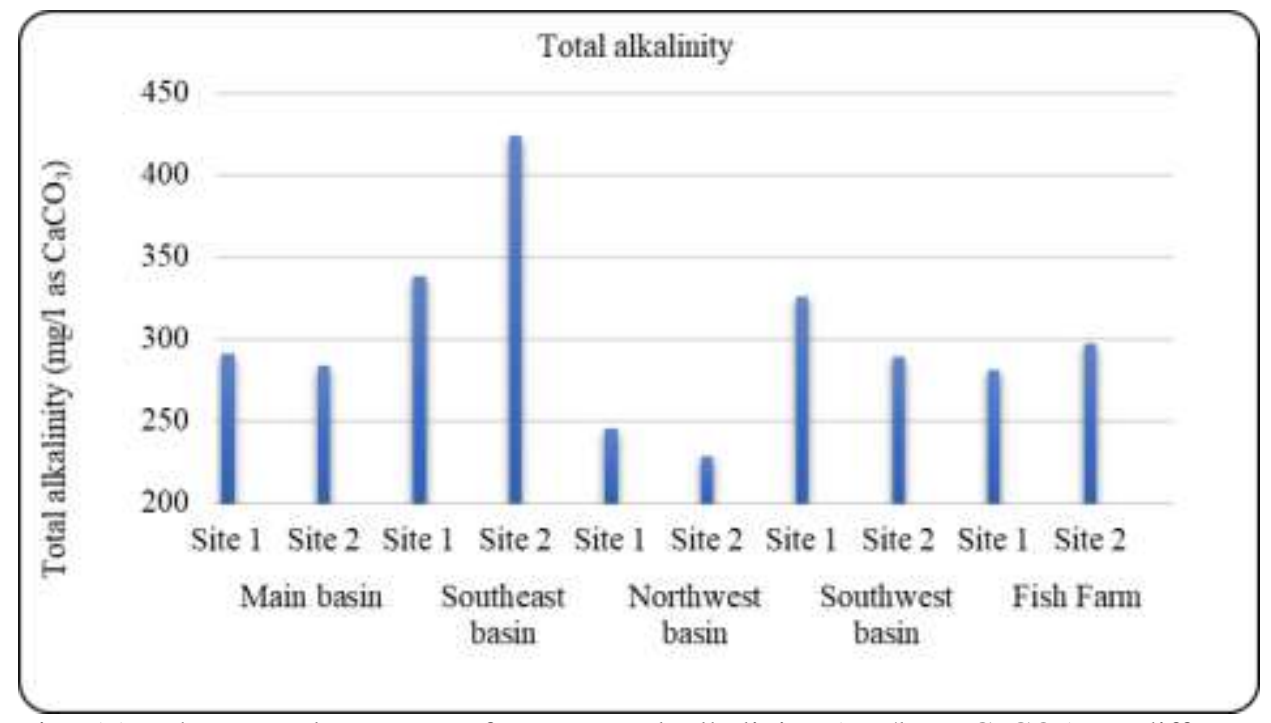

Fig. (7). The annual average of water total alkalinity $\left(\mathrm{mg} / \mathrm{l}\right.$ as $\left.\mathrm{CaCO}_{3}\right)$ at different sampling sites.

The highest value of total alkalinity in Mariout Lake could be attributed to the increased amount of organic matter accessible to bacterial decomposition resulted from different effluents discharged into the Lake which represented by increasing sewage, domestic, agricultural and industrial drainage water, and bicarbonate is the final product of the decomposition (Abdo and El-Nasharty, 2010). According to Wurts and 
Durborow (1992), alkalinity between 75 and $200\left(\mathrm{mg} / \mathrm{l}\right.$ as $\left.\mathrm{CaCO}_{3}\right)$, but not less than $20\left(\mathrm{mg} / \mathrm{l}\left(\mathrm{CaCO}_{3}\right)\right.$ is ideal in an aquaculture pond. Stone and Thomtrode (2004) suggested $50-150\left(\mathrm{mg} / \mathrm{l}\right.$ as $\left.\mathrm{CaCO}_{3}\right)$ as desirable range; while an acceptable range of above 20 and less than $400\left(\mathrm{mg} / \mathrm{l}\right.$ as $\left.\mathrm{CaCO}_{3}\right)$ for fishponds. Total alkalinity values greater than $300\left(\mathrm{mg} / \mathrm{l}\right.$ as $\left.\mathrm{CaCO}_{3}\right)$ are undesirable for fish growth and cause stress as previously mentioned by Bhatnagar and Devi (2013).

\section{Total hardness (TH):}

It is the measure of alkaline earth elements such as calcium and manganese, strontium, zinc, and hydrogen ions. Calcium and magnesium are essential to fish for metabolic reactions such as bone formation (Bhatnagar and Devi, 2013). TH values obtained during the present study ranged between $1250 \mathrm{mg} / \mathrm{l}$ as $\left(\mathrm{CaCO}_{3}\right)$ during autumn at (Main Basin at site 1) and $5400 \mathrm{mg} / \mathrm{l}$ as $\left(\mathrm{CaCO}_{3}\right)$ during spring at (Fish Farm at site 2). Fig. (8) showing that the annual average of total hardness values were between $1722.5\left(\mathrm{mg} / \mathrm{l}\right.$ as $\left.\mathrm{CaCO}_{3}\right)$ in (Southeast Basin) and 3235.083 $\left(\mathrm{mg} / \mathrm{l}\right.$ as $\left.\mathrm{CaCO}_{3}\right)$ in (Fish Farm).

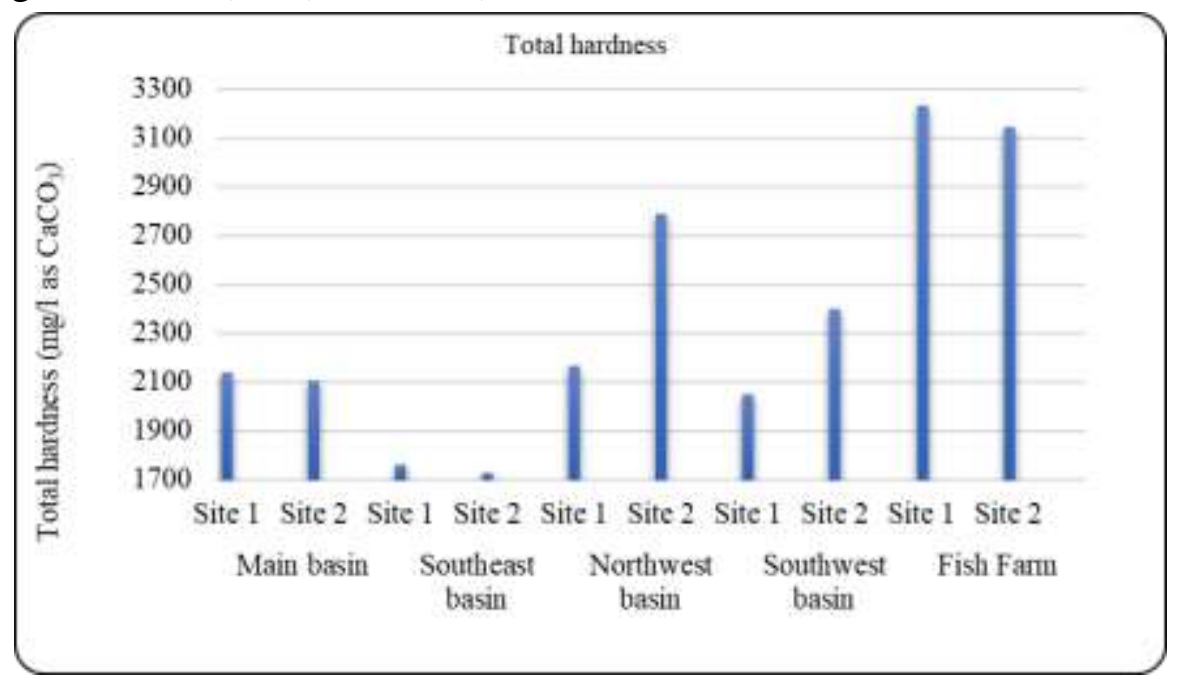

Fig. (8). The annual average of water total hardness $\left(\mathrm{mg} / \mathrm{l}\right.$ as $\left.\mathrm{CaCO}_{3}\right)$ at different sampling sites.

Similar values obtained by Farouk et al. (2020) who mentioned that $\mathrm{TH}$ values in (basin 6000) were between 1511.0 and $2534.4 \mathrm{mg} / \mathrm{l}$, while these values in (basin 1000) were between 1616 and $2193.3 \mathrm{mg} / \mathrm{l}$. The ideal value of hardness for fish culture is at least $20 \mathrm{ppm}$, as recommended by Swann (1997).

Nitrite $\left(\mathrm{NO}_{2}\right)$ : 
It is an intermediate product of the nitrification bacterial process, produced by the autotrophic Nitrosomonas bacteria combining oxygen and ammonia. Nitrite can be termed as an invisible killer of fish because it oxidizes hemoglobin to methemoglobin in the blood, turning the blood and gills brown and hindering respiration damage for nervous system, liver, spleen and kidneys of the fish (Bhatnagar and Devi, 2013). $\mathrm{NO}_{2}$ values obtained during the present study were between 0.02 and $0.2 \mathrm{mg} / \mathrm{l}$. Nitrite annual average was between 0.03 and $0.1225 \mathrm{mg} / \mathrm{l}$ in (Southeast Basin) and (Main Basin), respectively (Fig. 9).

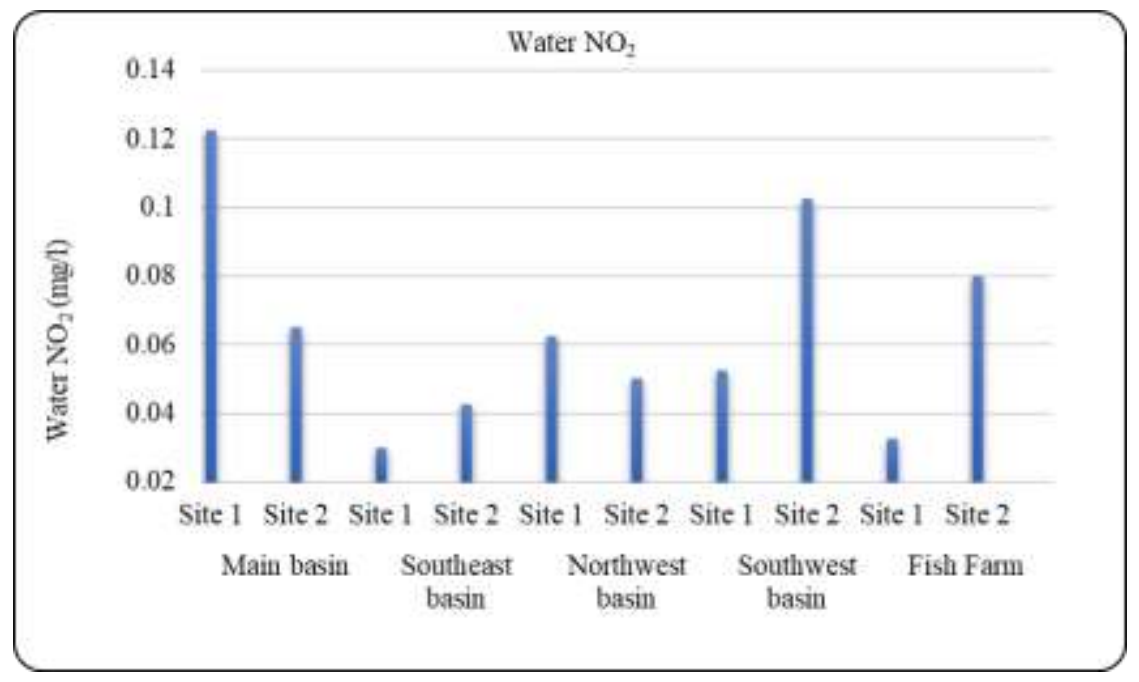

Fig. (9). The annual average of water nitrites ( $\mathrm{mg} / \mathrm{l})$ at different sampling sites.

Santhosh and Singh (2007) recommended nitrite concentration in water should not exceed $0.5 \mathrm{mg} / \mathrm{l}$. These values are within the standard safe limit as indicated by OATA (2008) who recommended that nitrite should not exceed $0.2 \mathrm{mg} / \mathrm{l}$.

Nitrate (NO3):

Nitrate is harmless and is produced by the autotrophic Nitrobacter bacteria combining oxygen and nitrite (Bhatnagar and Devi, 2013). $\mathrm{NO}_{3}$ values obtained during the study were between 0.05 during spring at (Northewest Basin and Main Basin at site 2) and $2.32 \mathrm{mg} / \mathrm{l}$, which recorded during summer at (Southeast Basin at site 1). $\mathrm{NO}_{3}$ annual average was between 0.15 and $1.115 \mathrm{mg} / \mathrm{l}$ in (Southern-west Basin and Fish Farm) and (Southeast Basin), respectively (Fig. 10). Furnas (1992) revealed that denitrification will be most intense where detritus accumulates and in water bodies subject to enhanced nutrient loading from pollution. 


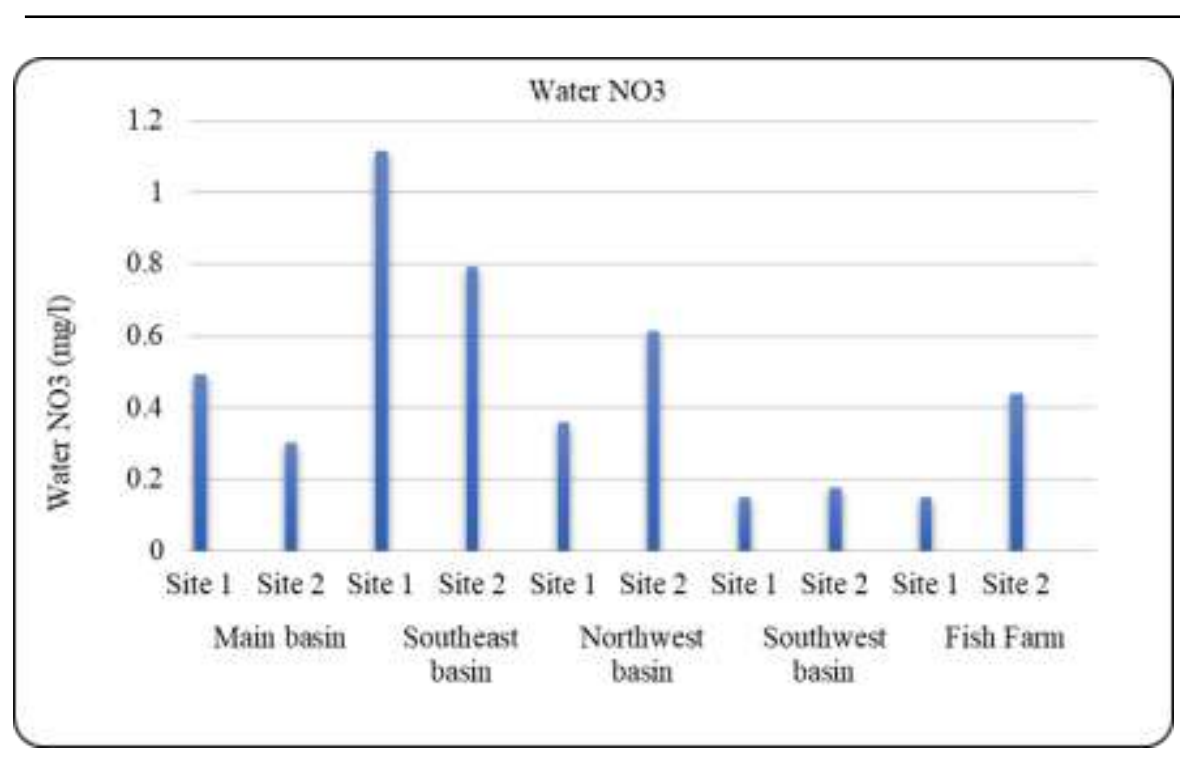

Fig. (10.) The annual average of water nitrates (mg/l) at different sampling sites.

Santhosh and Singh (2007) described the favorable range of $0.1 \mathrm{mg} / \mathrm{l}$ to $4 \mathrm{mg} / \mathrm{l}$ in fish culture water. The increased values of water $\mathrm{NO}_{2}$ and $\mathrm{NO}_{3}$ in some basins of the Lake, may be due to sewage effluents (Alnagaawy et al., 2018).

\section{Total nitrogen (TN):}

Nitrogen is an essential nutrient for plants and animals. However, an excess amount of nitrogen in a waterway may lead to low levels of dissolved oxygen and negatively alter various organisms' life. TN values ranged between $0.4 \mathrm{mg} / \mathrm{l}$ during winter at (Fish Farm at site 1) and 3.98 $\mathrm{mg} / \mathrm{l}$ during autumn at (Southwest Basin at site 2). Fig. (11) showing that the annual average of total nitrogen was between $0.94 \mathrm{mg} / \mathrm{l}$ in (Fish Farm) and $2.3325 \mathrm{mg} / \mathrm{l}$ in (Northwest Basin). These results in partial agreement with Farouk et al. (2020) who noted that, the highest TN values were recorded in basins of 3000 feddans which consider as an Industrial drainage, while the lowest value recorded in basins of 1000 feddans which received its water as a mix of (a drainage water and agricultural waste water). 


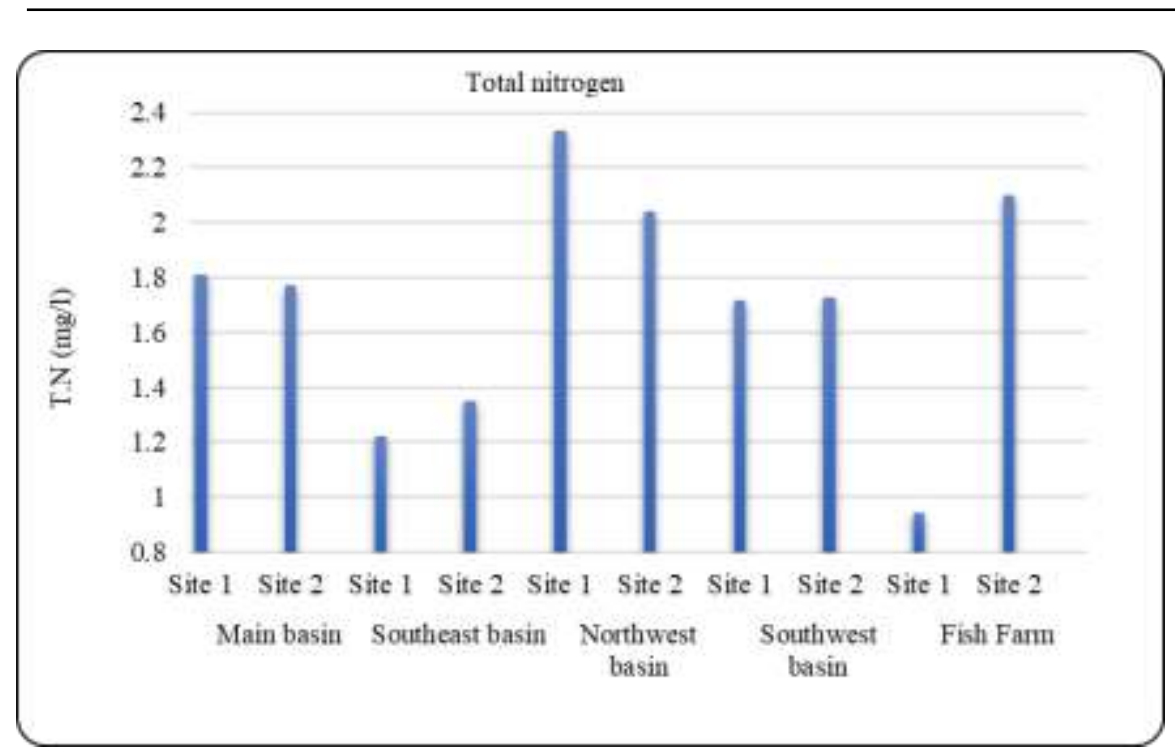

Fig. (11). The annual average of water total nitrogen (mg/l) at different sampling sites.

\section{Orthophosphates $\left(\mathrm{PO}_{4}\right)$ :}

It is the dissolved form of phosphorous, which is the available one for living organisms. Orthophosphates values that recorded during the present study ranged between $0.01 \mathrm{mg} / \mathrm{l}$ during winter at (Southeast Basin at site 1) and $0.42 \mathrm{mg} / \mathrm{l}$ during summer at (Northwest Basin at site 1). As shown in Fig. (12), the annual average of orthophosphates ranged between 0.045 and $0.31 \mathrm{mg} / 1$ in (Southeast Basin) and (Main Basin), respectively. ANZECC (2000) reported that the acceptable levels of $\mathrm{PO}_{4}$ is lower than $0.1 \mathrm{mg} / \mathrm{l}$ for freshwater and lower than $0.05 \mathrm{mg} / \mathrm{l}$ for marine water. Saad et $\boldsymbol{a l},(\mathbf{2 0 1 7})$ revealed that the higher concentrations of reactive phosphate are mostly due to the effect of drainage water enriched with phosphorous compounds.

\section{Total phosphorus (TP):}

Phosphorus is a limiting nutrient needed for the growth of aquatic plants and algae. However, excess concentrations especially in rivers and lakes can result to algal blooms. A lake with a concentration of below $0.01 \mathrm{mg} / \mathrm{l}$ is considered as oligotrophic, while concentrations between 0.01 and $0.02 \mathrm{mg} / \mathrm{l}$ are indicative of mesotrophy, and concentrations exceeding $0.02 \mathrm{mg} / \mathrm{l}$ are already considered eutrophic (Mueller and Helsel, 1999).

TP values obtained alongside the study were between 0.13 in summer (Fish Farm at site 1) and 0.92 in winter (Main Basin at site 2). Fig. (13) revealing that, the annual average of total phosphorous, which calculated 
at the end of the present study, was between 0.195 and $0.705 \mathrm{mg} / \mathrm{l}$ in (Fish Farm) and (Southeast Basin), respectively.

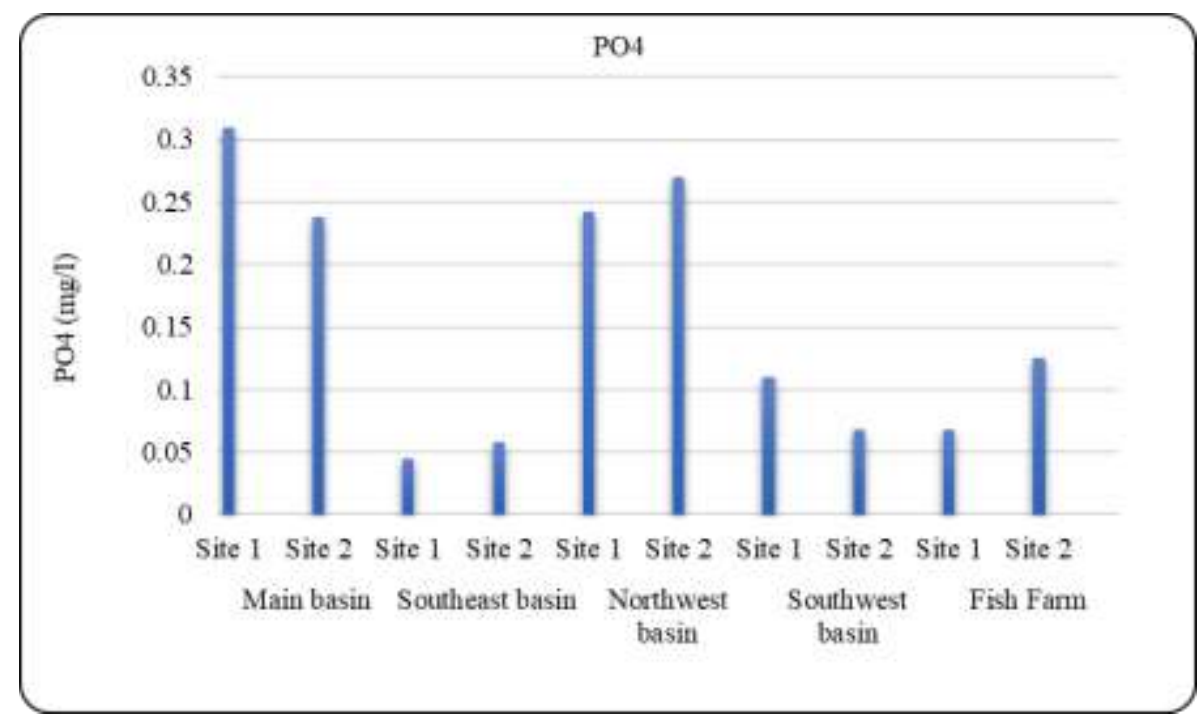

Fig. (12). The annual average of water orthophosphates (mg/l) at different sampling sites.

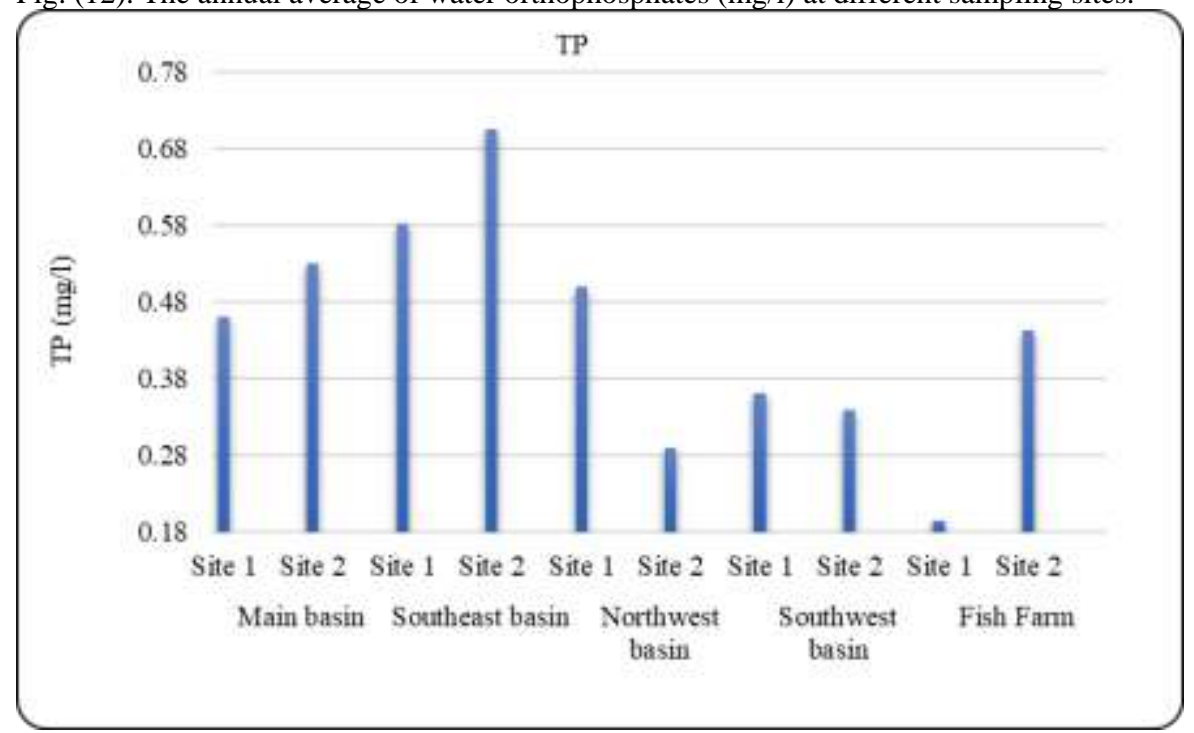

Fig. (13). The annual average of water total phosphorous (mg/l) at different sampling sites.

\section{Chlorophyll "a":}

Chlorophyll ' $a$ ' is a blue-green microcrystalline solid. It constitutes about 1 to $2 \%$ of the planktonic algae dry weight (Chernomorsky and Segelman, 1988). Chlorophyll "a" values which recorded alongside the study were between $47.6 \mu \mathrm{g} / 1$ during autumn and $459.6 \mu \mathrm{g} / \mathrm{l}$ during spring in (Main Basin at site 2) and (Northwest Basin at site 2), respectively. 
Similar result mentioned by Farouk et al. (2020) who revealed that, maximum values of chlorophyll "a" were recorded during spring season of the study which may be related to increase phytoplankton with increase nutrients of TN and TP.

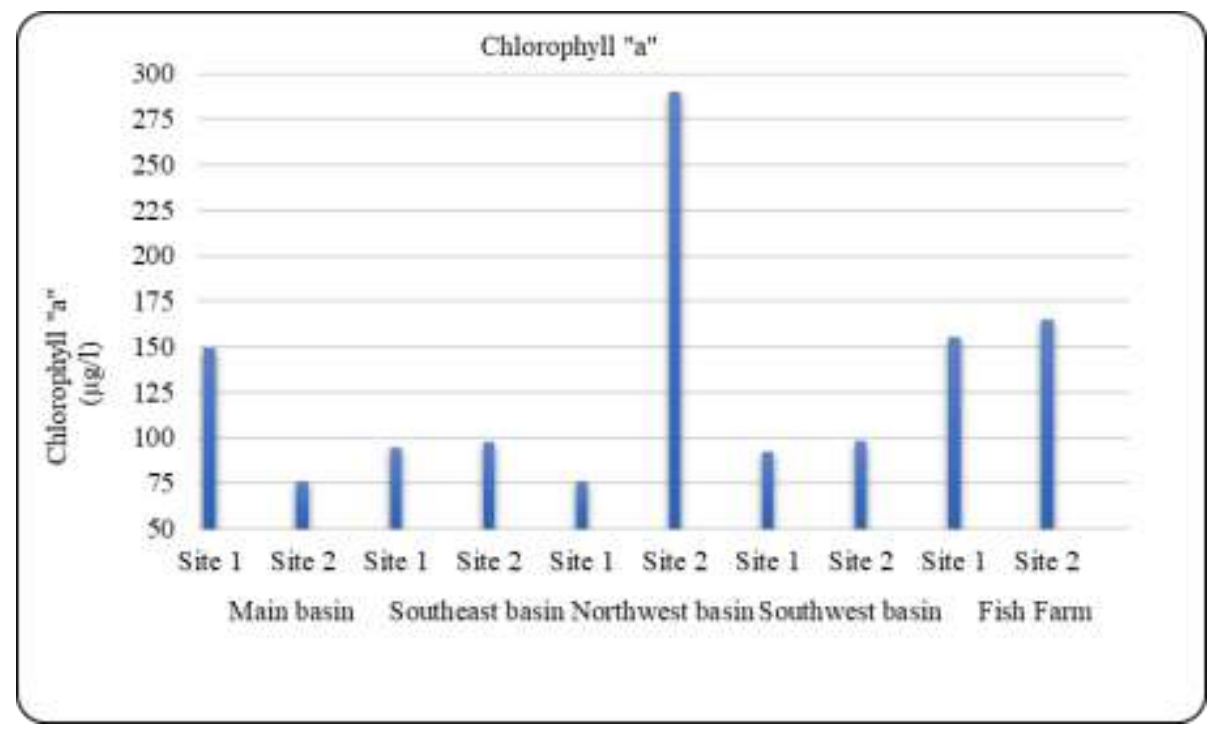

Fig. (14). The annual average of water chlorophyll "a" ( $\mu \mathrm{g} / \mathrm{l})$ at different sampling sites.

Fig. (14) indicating that the annual average of chlorophyll "a" recorded alongside the study period was between $75.65(\mu \mathrm{g} / \mathrm{l})$, which recorded in (Northern-west Basin) and $290.2(\mu \mathrm{g} / \mathrm{l})$ that recorded in (Main Basin). Depending on the obtained value of chlorophyll "a" Mariout lake classified as Hypertrophic, according to (O.E.C.D, 1982) who reported that lake water classified as hypertrophic when chlorophyll "a" values higher than $75(\mu \mathrm{g} / \mathrm{l})$.

\section{Conclusion and recommendation}

It could be concluded that the lake suffering from highly eutrophication, represented by critical values of some water quality characteristics, such as nutrients, which resulted from different effluents that discharged into the lake. Based on that, it is highly recommended to treat sewage, agricultural and industrial wastes dumping into the lake in order to improve its environmental status.

\section{References}

Abdo, M. H. and El-Nasharty, S. M. (2010). Physico-chemical evaluations and trace metals distribution in water-surficial sediment of Ismailia Canal, Egypt, Nature and Science, 8(5): 198-206. 
Alnagaawy, A. M.; Sherif, M. H.; Assy, G. M. and Shehata, A. S. (2018). Impact of industrial pollutants on some water quality parameters of Edku, Mariout lakes and the Nile River. Inter. J. Env., (7):1-15.

ANZECC, Australian and New Zealand Environment and Conservation Council \& Agriculture and Resource Management Council of Australia and New Zealand (ARMCANZ), 2000.

APHA (American Public Health Association), (2000). Standard methods for the examination of water and waste water $16^{\text {th }}$ ed.,Washington, D.C.

Bhatnagar, A. and Devi, P. (2013). Water quality guidelines for the management of pond fish culture. Internat. J. of Environ. Sc., 3(6): 1980-2009.

Chernomorsky, S. A. and Segelman, A. B. (1988). Biological activities of Chlorophyll derivatives. N. J. Med, 85, 669-73.

Davis, J. (1993). Survey of Aquaculture effluents permitting and 1993 standards in the South. Southern Regional Aquacult. Centre, SRAC publication no 465 USA, 4 PP.

Duncan, D. B. (1955). Multiple range and multiple F-tests. Biomet. 11: 115.

El-Rayis, O. A., Hemeda, E. I. and Shaaban, N. A. (2019). Steps for rehabilitation of a Lake suffering from intensive pollution; Lake Mariut as a case study. Egy. J. Aqua. Biol. \& Fisher., 23(2): 331-345.

El-Sherif, M. S. and El-Feky A. M. (2009). Performance of Nile tilapia (O. niloticus) fingerlings. II. Influence of different water temp. Int. $J$ Agric. Biol., 11:301-305.

Farouk, A., Abdel-Hamid, E. A. A. and Mekawy, M. T. (2020). Environmental Studies on Water Quality, Plankton and Bacterial ommunity in Mariout Lake, Egypt Egyptian Journal of Aquatic Biology \& Fisheries, Zoology Department, Faculty of Science, Ain Shams Univ., Cairo, Egypt. Vol. 24(4): 139 - 158.

Francis-Floyd, R. (2003). Dissolved Oxygen for Fish Production, Fact Sheet FA 27, The Department of Fisheries and Aquaculture, Florida Cooperative Extension Service, Institute of Food and Agricultural Sciences, University of Florida.

Furnas, M. J. (1992). The behavior of nutrients in tropical aquatic ecosystems. p. 29-68. In: Connell, D.W. and D.W. Hawker (eds.). Pollution in Tropical Aquatic Systems. CRC Press Inc., London, U.K. 
Gehan M. El Zokm, Hermine R. Z. Tadros, Mohamed A. Okbah and Galila H. Ibrahim (2018). Eutrophication assessment using TRIX and Carlson's indices in Lake Mariout Water, Egypt. Egyptian Journal of Aquatic Biology and Fisheries Vol. 22(5): 321-339.

Mateo, M. A. (2009). Lake Mariut: An ecological assessment. WADI project water demand integration; INCO-CT-2005-015226.

Mitchell, M. K. and Stapp, W. B. (1992). Field Manual for Water Quality Monitoring, an environmental education program for schools. GREEN: Ann Arbor, MI.

Mueller, D. K. and Helsel, D. R. (1999). Nutrients in the Nation's WatersToo Much of a Good Thing? US. Geological Survey Ciricular 1136. National WQ Assessment Program.

O.E.C.D., Organization for Economic Cooperation and Development, (1982). Eutrophication of Waters, Monitoring, Assessment and Control. Paris, OECD.

Ornamental Aquatic Trade Association (OATA) (2008). Water Quality Criteria-Ornamental Fish. Company Limited by Guarantee and Registered in England No. 2738119 Registered Office Wessex House, 40 Station Road, Westbury, Wiltshire, BA13 3JN, UK.

Phang, S. M. (1991). Role of Algae In Livestock-Fish Integrated Farming Systems, Integrated Livestock-Fish Production System, Proceedings of the FAO/IPT, Institute of Advanced Studies, University of Malaya, Kuala Lumpur, Malaysia.

Richlen, M. L., Morton, S. L., Jamali, E. A., Rajan, A. and Anderson, D. M. (2010). The catastrophic 2008 - 2009 red tide in the Arabian Gulf region, with observations on the identification and phylogeny of the fish-killing dinoflagellate Cochlodinium polykrikoides. Harmful Algae, 9: 163-172.

Saad, A. S., Massoud, R. A. and Ghorab, M. A. (2017). Assessment of the Physicochemical Characteristics and Water Quality Analysis of Mariout Lake, Southern of Alexandria, Egypt. J Environ Anal Toxicology, 7(1): 1-19.

Santhosh, B and Singh, N. P. (2007). Guidelines for water quality management for fish culture in Tripura, ICAR Research Complex for NEH Region, Tripura Center, Publication No.29.

SAS. (2009). Statistics. SAS Institute Inc. editors, Cary, NC.

Scapini, F. (2009). Sustainable management of Mediterranean coastal fresh and transitional water bodies: a socio-economic and 
environmental analysis of changes and trends to enhance and sustain stakeholder benefits. Coastal Water Bodies Nature and Culture Conflicts in the Mediterranean 1-5.

Stone, N. M. and Thomforde, H. K. (2004). Understanding Your Fish Pond Water Analysis Report. Cooperative Extension Program, Univ. of Arkansas at Pine Bluff Aquaculture / Fisheries.

Swann, L. D. (1997). A Fish Farmer Guide to Understanding Water Quality, Aquaculture Extension Illionis, Purdue University, Indiana Sea Grant Program Fact Sheet, AS-503.

Thompson, M., Brandes, D. and Kney, A. (2012). Using electronic conductivity and hardness data for rapid assessment of stream water quality. J. Environ. Manage, 104: 152-157.

Tucker, C. S. (1984). Carbon dioxide. in T.L. Wellborn, Jr. and J.R. MacMillan (eds.) For Fish Farmers 84-2. Mississippi Co-operative Extension Service

Vollenweider, R. A. (1969). A manual on methods for measuring primary production in aquatic environments. IBP Handb. No. 12, Blackwell Sci. Publ., Oxford., 213 pp.

Wurts, W. A. and Durborow, R. M. (1992). Interactions of pH, Carbon Dioxide. Alkalinity and Hardness in Fish Ponds. Southern Regoinal Aquaculture Center, SRAC Puplication No. 464. 


\section{تأثير الملوثات المختلفة علي بعض عوامل جودة المياه لبحيرة مريوط - مصر

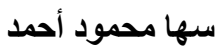

قسم الليمينولوجى، المعمل المركزى لبحوث الثروة السمكية، مركز البحوث الزراعية

$$
\text { الملخص العربي ليحوث الثرون }
$$

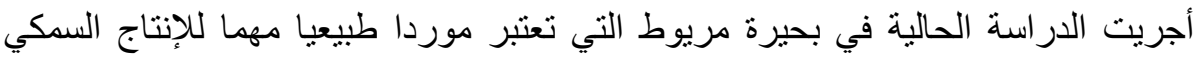

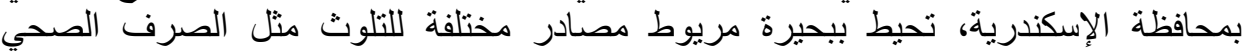

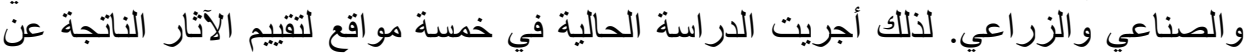

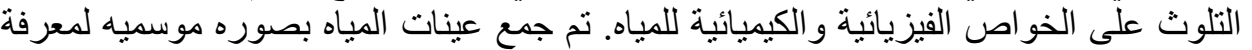

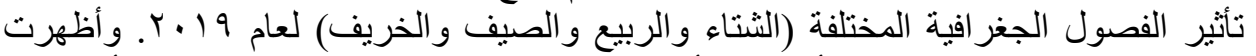

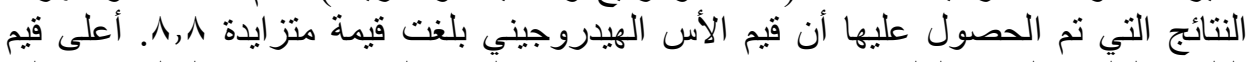

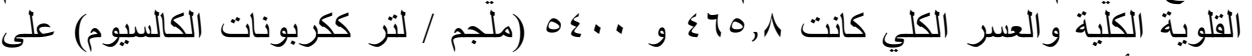

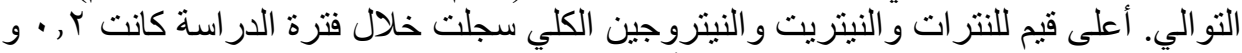

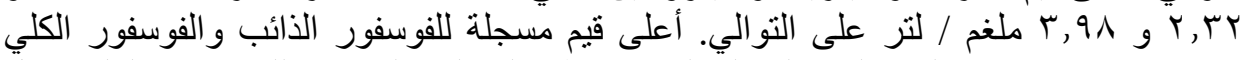

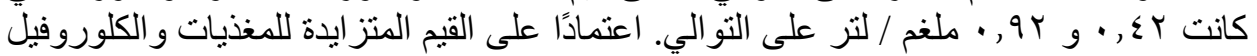

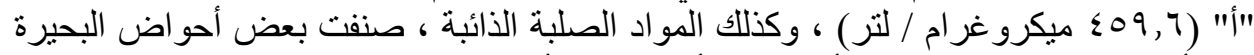

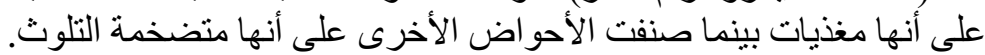

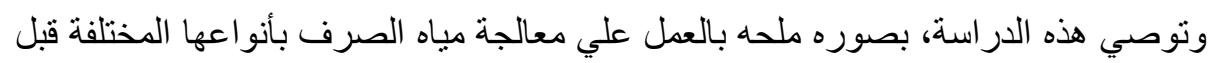
تصريفها إلي البحيرة لتقليل معدلات التلوث وتحسين صفات جودة العياه بلهي التهاه 